\title{
Invadopodia: proteolytic feet of cancer cells
}

\author{
Gizem BATI $^{1}$, Devrim PESEN OKVUR ${ }^{2 * *}$ \\ ${ }^{1}$ Graduate Program in Biotechnology and Bioengineering, İzmir Institute of Technology, İzmir, Turkey \\ ${ }^{2}$ Department of Molecular Biology and Genetics, İzmir Institute of Technology, İzmir, Turkey
}

Received: 29.04 .2014

- $\quad$ Accepted: 21.07.2014

- Published Online: 24.11 .2014

- $\quad$ Printed: 22.12 .2014

\begin{abstract}
The leading cause of death in cancer patients is metastasis. Invasion is an integral part of metastasis and is carried out by proteolytic structures called invadopodia at the cellular level. In this introductory review, we start by evaluating the definition of invadopodia. While presenting the upstream signaling events involved, we integrate current models on invadopodia. In addition, we discuss the significance of invadopodia in $2 \mathrm{D}$ and $3 \mathrm{D}$ and in vivo. We finally point out technical challenges and conclude with open questions in the field.
\end{abstract}

Key words: Metastasis, invadopodia

\section{Metastasis}

The leading cause of death in cancer patients is metastasis. Metastasis defines both the process of spreading of cancer cells from the primary tumor and the resulting secondary tumors. The primary tumor changes its place (meta + statis) and new tumors form at distant sites. During metastasis of carcinoma (cancer of epithelial tissue), tumor cells proliferate in an uncontrolled fashion, induce angiogenesis (new blood vessel formation), degrade the underlying basement membrane and penetrate into the connective tissue, migrate towards blood vessels, intravasate (enter blood vessels), survive in the blood circulation, extravasate (exit blood vessels), and form secondary tumors in distant organs (Figure 1A). Therefore, cancer metastasis is a disease of altered cell adhesion, motility, and invasion.

\section{Definition of invadopodia}

Under physiological conditions such as bone resorption, cells invade into tissues in a tightly regulated manner. Normal bone osteoclasts form special cellular structures called podosomes to degrade and thus remodel the bone matrix. During cancer metastasis, tumor cells perform uncontrolled invasion using cellular structures called invadopodia (Figure 1B). The term invadopodia was first used by Chen (1989) to describe membrane protrusions involved in the local degradation of the extracellular matrix. After 25 years, the field has grown to be complex and rather complicated even in terms of definitions. There are 3 major structures in cells, each of which can be defined

* Correspondence: devrimpesen@iyte.edu.tr in terms of their molecular components and the functions they carry out. These are focal adhesions, podosomes, and invadopodia. They do have similarities, but they are also distinct from one another. In attempts to clear up some of the confusion, podosomes and invadopodia have also been collectively called invadosomes. Although focal adhesions do share common protein markers with podosomes, they were thought to be more distinct from podosomes and invadopodia; however, recently proteolytic activity has also been observed for these structures, further blurring the borders between the definitions of these structures (McNiven, 2013). Available data raise the question of whether focal adhesions, podosomes, and invadopodia share a common precursor. A conservative comparison of focal adhesions, podosomes, and invadopodia is presented in the Table. Please note that this table is compiled conservatively to include the data most commonly agreed upon in the literature. There are also several reviews and milestone papers describing in detail various aspects summarized here (Ayala et al., 2006; Gimona and Buccione, 2006; Linder, 2007; Gimona et al., 2008; Caldieri et al., 2009; Linder, 2009; Yilmaz and Christofori, 2009; Linder et al., 2011a, 2011b; Murphy and Courtneidge, 2011; Oser et al., 2011; Yamaguchi, 2012). In particular, there are comprehensive reviews on the signaling mechanisms involved (Stylli et al., 2008; Destaing et al., 2011; Hoshino et al., 2013). In this review, we will focus on invadopodia, integrate current models, and point out technical challenges and open questions in the field. 


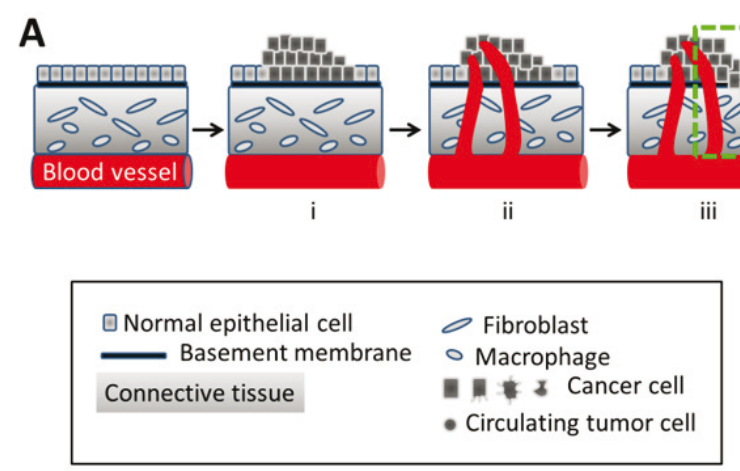

B

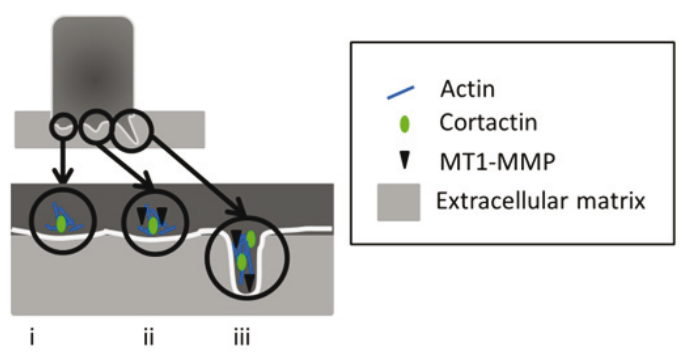

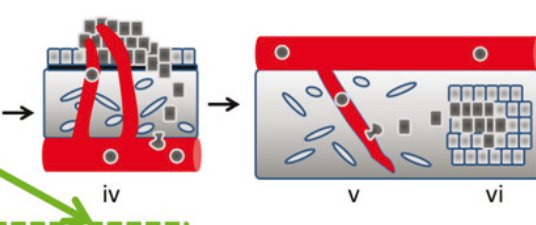

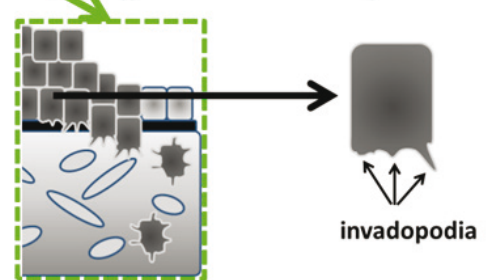

C

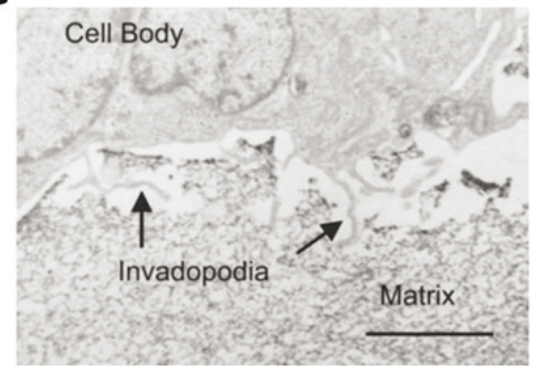

Figure 1. Metastasis and invadopodia. A. Metastasis comprises (i) uncontrolled proliferation, (ii) angiogenesis, (iii) invasion, (iv) intravasation, (v) extravasation, and (vi) secondary tumor formation. Invasion involves matrix degradation carried out by invadopodia. B. Invadopodiaform and mature at multiple stages: (i) initiation, (ii) stabilization, and (iii) maturation (see also Figure 3). Initiation involves recruitment of actin and cortactin, MT1-MMP recruitment leads to stabilization. Maturation stage involves matrix degradation. When cells are on a thick matrix, invadopodia appear as membrane protrusions penetrating into the extracellular matrix. C. Electron micrograph of an MDA-MB-231 cell cultured on gelatin. The ultrastructure of invadopodia (arrows) is shown. Reprinted by permission from Macmillan Publishers Ltd: Oncogene (Bowden et al., 1999) copyright 1999.

Invadopodia are relatively dynamic, actin rich, proteolytic cellular structures formed by invasive cancer cells (Bowden et al., 2006; Buccione et al., 2009; Linder, 2009; Linder and Aepfelbacher, 2003) (Figure 2). Invadopodia can be from a few hundred nanometers to several microns wide and can be up to 8 micrometers if the underlying matrix is thick enough (Baldassarre et al., 2003). Invadopodia also form on stiff substrates such as glass and thus they can be studied with high resolution imaging (DesMarais et al., 2009). The molecular markers for invadopodia include actin and its associated proteins cortactin, Arp2/3, N-WASP, Nck1, and cofilin as well as matrix metalloproteinase MT1-MMP (Artym et al., 2006; Stylli et al., 2008; Oser et al., 2009, 2011). In addition, actin filaments, microtubules, and intermediate filaments cooperate during invadopodia elongation (Schoumacher et al., 2010). In melanoma cells, invadopodia contain $\alpha 3 \beta 1$ integrin at the core and $\alpha 5 \beta 1$ integrin at the periphery (Mueller et al., 1999). Integrins at invadopodia may function to signal and to focus degradation of the extracellular matrix (ECM) (Buccione et al., 2009; Mueller et al., 1999). However, it is unclear if invadopodia have an adhesive function as they lack vinculin (Gimona et al., 2008; Linder, 2009). That is, whether invadopodia require local adhesion at the sites of formation is unknown. Preliminary results from our lab using nano-patterned surfaces suggest that invadopodia do not require local adhesion (unpublished data). Experiments using nanoand micro-patterned substrates can present valuable approaches to answer such questions and other aspects of invadopodia/podosome research such as dynamics of mechanical properties (Labernadie et al., 2010).

In images of cells forming invadopodia, the Golgi complex appears to be polarized and juxtaposed to the site of invadopodia, suggesting a link between proteolytic activity and membrane transport (Baldassarre et al., 2003; Buccione et al., 2009; Caldieri and Buccione, 2010). However, if and how the spatial positioning of invadopodia is controlled is not known. In addition, the position and orientation of the Golgi can be modulated by micrometer scale surface patterns (Thery et al., 2006). Therefore, if invadopodia are positionally linked to the 
Table. Comparison of focal adhesions, podosomes, and invadopodia.

\begin{tabular}{llll}
\hline & Focal adhesions & Podosomes & Invadopodia \\
\hline Cell type & virtually all cells & $\begin{array}{l}\text { osteoclasts, macrophages, } \\
\text { endothelial cells, } \\
\text { smooth muscle cells }\end{array}$ & cancer cells \\
Function & $\begin{array}{l}\text { adhesion, } \\
\text { matrix degradation? }\end{array}$ & matrix degradation & matrix degradation \\
Cellular localization & cell periphery & distributed & leading edge and proximal to Golgi \\
& Actin & & Actin \\
& Vinculin & Actin & Arp2/3 \\
& Talin & Vinculin & Cortactin \\
Composition & Paxillin & WASP & N-WASP \\
& Focal adhesion kinase & Grb2 & MT1-MMP \\
Integrin & & Cofilin \\
Shape & ellipse & ring 5 & MT1-MMP \\
Size & $<20 \mu \mathrm{m}$ & $<1 \mu \mathrm{m} \times 4 \mu \mathrm{m}$ & dot \\
Number per cell & $<400$ & $20-100$ & $<8 \mu \mathrm{m} \times 5 \mu \mathrm{m}$ \\
Stability/ Persistence & stable/several hours & highly dynamic/2-12 min & dynamic/up to $3 \mathrm{~h}$ \\
\hline
\end{tabular}

Golgi, changing the position of the Golgi by culturing cells on different micrometer scale surface patterns should also change the localization of invadopodia. Thus micropatterned substrates present themselves as valuable tools for the question at hand.

\section{Upstream of invadopodia}

Growth factors act as intercellular signaling molecules that promote various processes such as cell growth, proliferation, differentiation, and motility. In addition, growth factor receptors and integrins are known to crosstalk (Moro et al., 2002; Yamada and Even-Ram, 2002; Alam et al., 2007; Gilcrease, 2007). Growth factors can be soluble, transmembrane, or ECM bound proteins (Ruoslahti et al., 1992; Massague and Pandiella, 1993; Taipale and Keski-Oja, 1997). Epidermal growth factor (EGF) is 1 of the 7 ligands of EGF receptor (EGFR also known as ErbB1), which is in turn the most studied member of the ErbB receptor family (Cohen, 1962; Carpenter and Cohen, 1990; Harris et al., 2003; Singh and Harris, 2005). Furthermore, EGFR expression correlates with poor prognosis in breast cancer (Sainsbury et al., 1985; Lewis et al., 1990; Memon et al., 2006). EGF is known to induce motility and invadopodia formation in breast cancer cells (Yamaguchi et al., 2005). However, whether EGFR is present at invadopodia and acts directly and locally or not is not known.

In terms of signal transduction, growth factor receptor tyrosine kinase and integrin initiated upstream events have been shown to promote invadopodia formation through phosphorylation of cortactin via a Src and Arg dependent pathway (Stylli et al., 2008; Oser et al., 2010; Destaing et al., 2011; Mader et al., 2011; MacGrath and Koleske, 2012). b1 integrin has been shown to promote metastasis, invadopodia maturation, and matrix degradation through Arg (Beaty et al., 2013). Local changes in $\mathrm{pH}$ induced by NHE1 are also shown to regulate cortactin phosphorylation (Magalhaes et al., 2011). Furthermore, small GTPases are shown to be spatiotemporally regulated at invadopodia where $\mathrm{RhoC}$ is inactivated at the center of invadopodia and is activated at its periphery so that cofilin is active at the center and is inactive at the periphery (Bravo-Cordero et al., 2011, 2012). Here, RhoC is shown to act through ROCK, which phosphorylates LIMK, which in turn phosphorylates and inactivates cofilin.

\section{An integrated model of invadopodia}

Over the years, valuable research has produced models that describe invadopodia. An integrated model is presented in Figure 3. One of the early studies classified invadopodia formation into 4 stages: I. Invadopodia initiation, II. Preinvadopodia, III. Mature invadopodia, and IV. Late invadopodia. Cortactin levels are at their maximum at stages II and III and subside afterwards, while actin levels reach a peak at stage III. MT1-MMP reaches a maximum at stage II and is stable thereafter, while matrix degradation saturates at stage III (Artym et al., 2006).

Later on, a more detailed model was presented by Oser et al. (2009), pointing out the central role of cortactin in invadopodia. Here, cortactin was shown to regulate cofilin and N-WASP activities and thus control the stages 

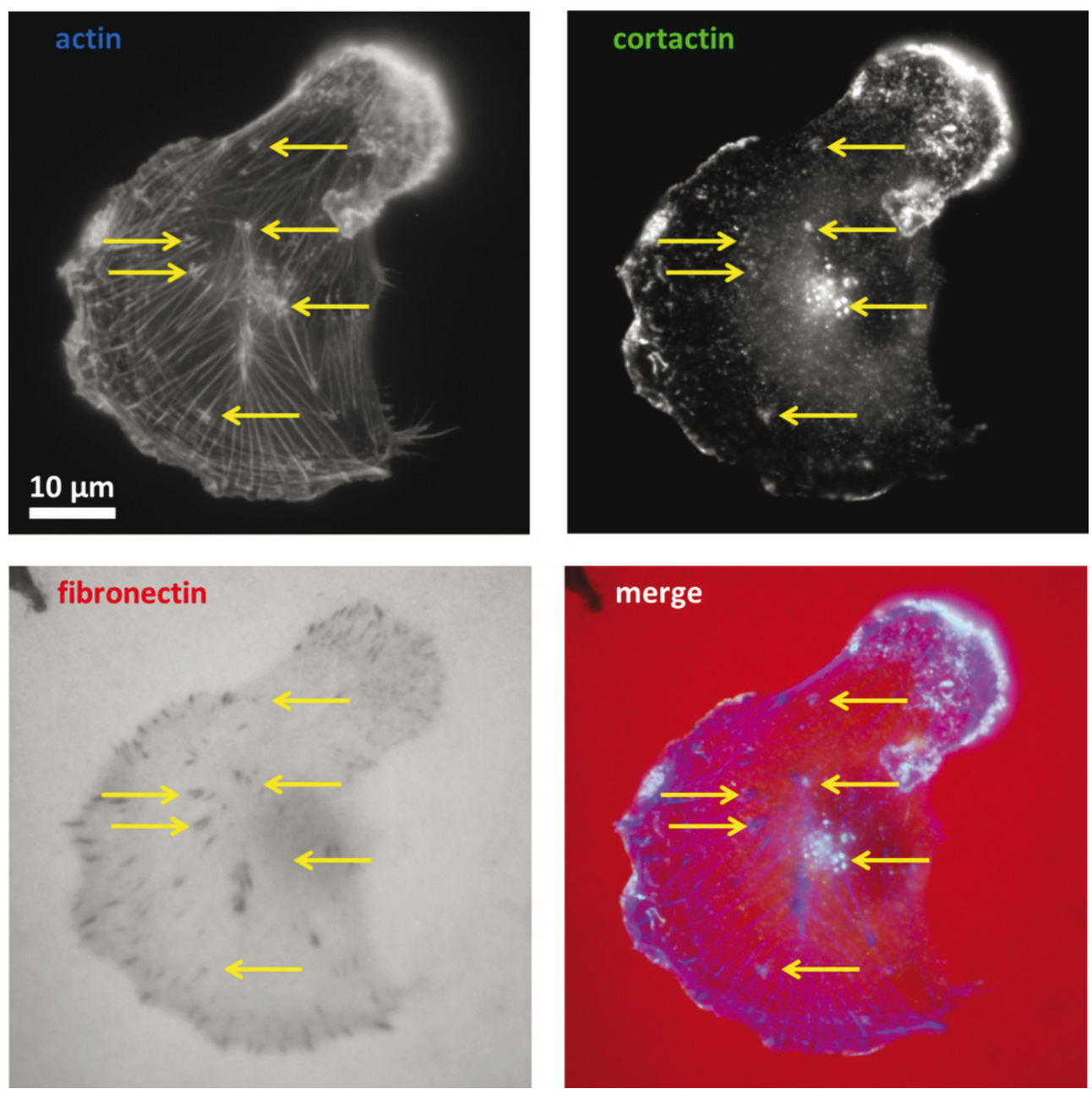

Figure 2. Immunofluorescence images of invadopodia in MDA-MB-231 breast cancer cells cultured on fibronectin, an extracellular matrix protein. Actin, cortactin, and fibronectin were labeled using blue-fluorescent phalloidin, cortactin specific antibodies followed by green-fluorescent secondary antibodies, and fibronectin specific antibodies followed by red-fluorescent secondary antibodies, respectively. Yellow arrows point to invadopodia. Cortactin and actin colocalize at invadopodia. At mature invadopodia, the underlying matrix of fibronectin is also degraded. In the merged image, cortactin, actin, and fibronectin are shown in green, blue, and red, respectively.

of invadopodia formation. Four stages were redefined here: Stage I - Precursor formation: Cortactin, N-WASP, cofilin, and Arp2/3 form a complex. Stage II - Activation of actin polymerization: Nck1 joins the precursor complex while phosphorylation of cortactin activates cofilin's severing activity, which in turn provides free barbed ends for Arp $2 / 3$ for new actin polymerization. Stage III - Stabilization: Cortactin is dephosphorylated, cofilin re-joins the complex, and invadopodia precursors are stabilized. Stage IV - ECM degradation: MT1-MMP is recruited to invadopodia and ECM is degraded.

The model by Oser et al. was then refined in terms of involvement of Tks5 and SHIP2, which are shown to be required for invadopodia maturation but not initiation
(Sharma et al., 2013). The integrated model we present here comprises 3 stages: initiation, stabilization, and maturation. Initiation here describes a combination of the previously described stages I and II and involves structural complex formation and actin polymerization. Stabilization includes MMP recruitment. At the maturation stage, MMPs are activated and matrix degradation takes place.

To recapitulate, the first events in the signal transduction pathways that result in invadopodia formation are integrin and/or growth factor activation. Although the intermediates are not entirely known, activation of $\mathrm{Src}$ is the key event for invadopodia formation. Src in turn activates Arg, which phosphorylates cortactin. While unphosphorylated cortactin, unphosphrylated 

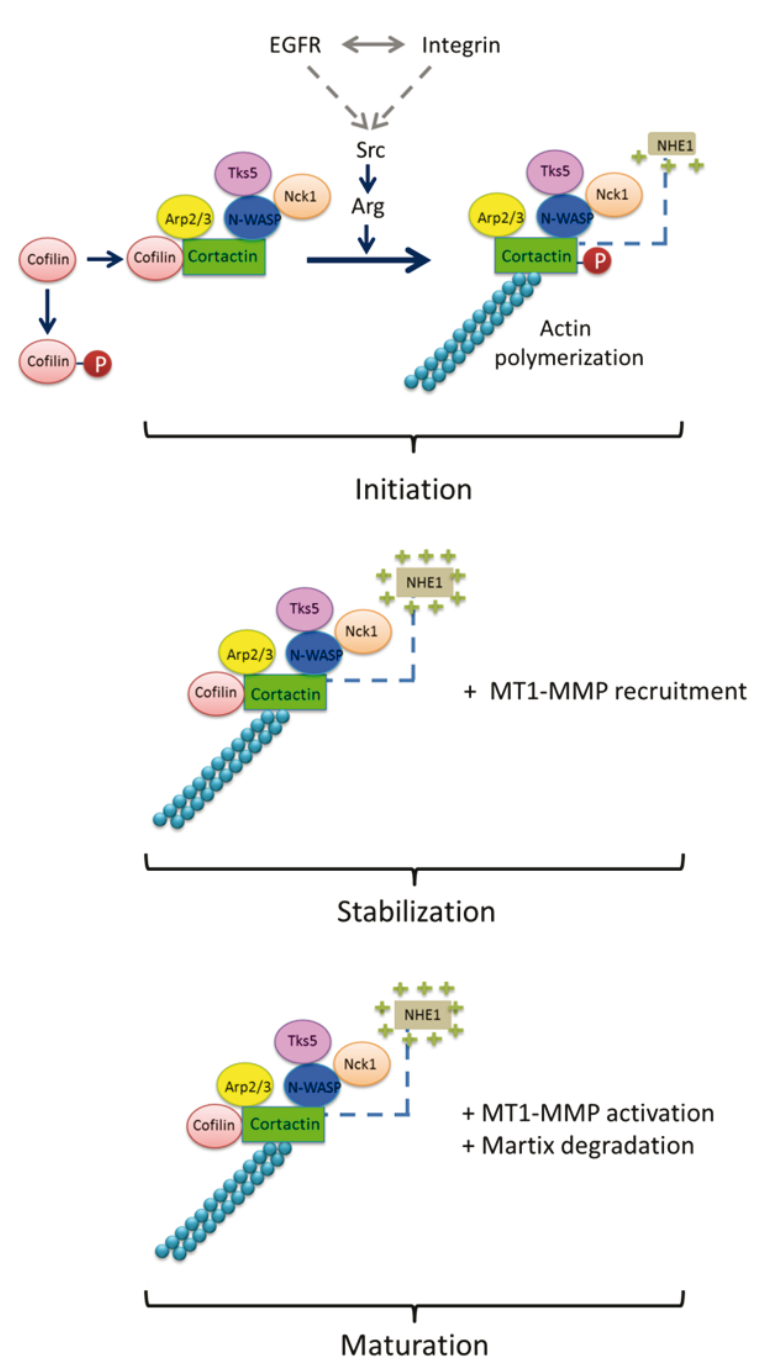

Figure 3. Integrated model for the initiation, stabilization, and maturation of invadopodia. Presented is a combination of various models published in the literature through the years. Initiation: Growth factor and/or integrin initiated signaling cascades result in phosphorylation of cortactin by Arg, which in turn is activated by Src. Phosphorylation of cortactin releases cofilin from the invadopodial complex comprising Arp2/3, N-WASP, Nck1, and Tks5. Release of cofilin promotes actin polymerization. Stabilization: Cortactin is dephosphorylated and cofilin is rerecruited to the complex. NHE1 induces local decrease in $\mathrm{pH}$ and MT1-MMP is recruited. Maturation: MT1-MMP is activated and matrix degradation takes place.

cofilin, N-WASP, Tks5, Nck1, and Arp2/3 coexist in the pre-initiation complex, after phosphorylation of cortactin, cofilin leaves the complex and enables actin polymerization. At the same time, NHE1, which causes a decrease in the local $\mathrm{pH}$, is recruited. During stabilization, cofilin is dephosphorylated and binds back to cortactin, and MT1-MMP is recruited to invadopodia. Finally, in the maturation stage MT1-MMP is activated and matrix degradation takes place.

\section{Invadopodia in $2 \mathrm{D}$ and $3 \mathrm{D}$ and in vivo}

Invadopodia are observed in both $2 \mathrm{D}$ and $3 \mathrm{D}$ cell cultures in vitro. Most research has been carried out in 2D cell culture, while studies in 3D conditions are increasing. In $2 \mathrm{D}$ cell culture, invadopodia are found at the ventral surface of cells. They even form on substrates such as glass without a matrix coating. If the substrate underneath cancer cells is degradable and thick enough, invadopodia extend as proteolytic protrusions into the matrix. When cancer cells are embedded in a 3D matrix, the definition of a ventral surface dissolves and cancer cells can form proteolytic protrusions at various points. As in $2 \mathrm{D}$, how the cellular localization of invadopodia is determined in $3 \mathrm{D}$ is unknown. However, invadopodia are composed of actin, cortactin, cofilin, Tks5, and MT1-MMP in both 2D and 3D cultures and in vivo settings (Blouw et al., 2008; Lizarraga et al., 2009; Magalhaes et al., 2011; Gligorijevic et al., 2012; Yu Machesky, 2012).

Invadopodia have been observed in $2 \mathrm{D}$ and $3 \mathrm{D}$ in vitro settings. However, the in vivo and physiological relevance has only been recently clarified. N-WASP expression is shown to increase in invasive breast cancer (Yu et al., 2012). Expressions levels of cortactin have been positively correlated with aggressiveness of head and neck squamous cell carcinoma and breast carcinoma (Buday and Downward, 2007; Clark et al., 2009). Expression level of Tks5 has been shown to increase in breast cancer and glioma (Seals et al., 2005; Stylli et al., 2012). MMP expression levels are known to be differentially regulated in various cancers (Kessenbrock et al., 2010). Early MMP inhibitors failed in clinical trials, and their nonproteolytic functions are speculated to be one of the reasons. However, there are still promising candidates such as MT1-MMP (Chen et al., 1994; Chen, 1996; Sabeh et al., 2009). In addition, upstream players that induce invadopodia formation such as Mena ${ }^{\mathrm{INV}}$, Arg, IL-6, EGFR, and faciogenital dysplasia protein Fgd1, are known to have increased expression levels in various cancers (Ayala et al., 2009; Clark et al., 2009; Li et al., 2010b; Wang et al., 2004, 2007; Gil-Henn et al., 2013). Thus both upstream regulators and structural components of invadopodia present vital opportunities for diagnosis and therapy.

\section{Technical bottlenecks for research on invadopodia}

A technical limitation for research on invadopodia has been the limited number of assays for proteolytic activity. Fluorescently labeled gelatin or fibronectin is commonly used in addition to DQ-collagen, which becomes fluorescent upon degradation. In addition, Packard et al. (2009) have used a substrate that shows sites of degradation 
by MT1-MMP. Fluorogenic peptide substrates have also been utilized to assay matrix degradation by MMPs (Leight et al., 2013). The field would greatly benefit from novel assays that allow the determination of matrix degradation, particularly for 3D culture and in vivo settings.

Another bottleneck has been the difficulty in analyzing invadopodia in a quantitative manner. Counting invadopodia in a cell or in a field of view based on colabeling of actin and cortactin, for instance, requires either brute force of manual counting or elegant image processing approaches. An alternative approach has been to quantify the area of matrix degraded by invadopodia rather than counting individual structures ( $\mathrm{Li}$ et al., 2010a). Welldesigned image processing approaches would be greatly beneficial for research on invadopodia.

\section{Conclusions and open questions}

In conclusion, while our understanding of invadopodia continues to improve despite confusion even at the definitions level, the field requires the incorporation of new technologies and there are many open questions waiting to be answered, such as: Do focal adhesions, podosomes, and invadopodia share a common precursor? Do invadopodia have an adhesive function? How is the cellular localization of invadopodia controlled? Is EGFR present at invadopodia and does it act directly or indirectly? How can we better assay the proteolytic activity of invadopodia? How can we improve the quantitative analysis of invadopodia? How

\section{References}

Alam N, Goel HL, Zarif MJ, Butterfield JE, Perkins HM, Sansoucy BG, Sawyer TK, Languino LR (2007). The integrin-growth factor receptor duet. J Cell Physiol 213: 649-653.

Artym VV, Zhang Y, Seillier-Moiseiwitsch FO, Yamada KM, Mueller SC (2006). Dynamic interactions of cortactin and membrane type 1 matrix metalloproteinase at invadopodia: Defining the stages of invadopodia formation and function. Cancer Res 66: 3034-3043.

Ayala I, Baldassarre M, Caldieri G, Buccione R (2006). Invadopodia: A guided tour. Eur J Cell Biol 85: 159-164.

Ayala I, Giacchetti G, Caldieri G, Attanasio F, Mariggio S, Tete S, Polishchuk R, Castronovo V, Buccione R (2009). Faciogenital dysplasia protein Fgd1 regulates invadopodia biogenesis and extracellular matrix degradation and is up-regulated in prostate and breast cancer. Cancer Res 69: 747-752.

Baldassarre M, Pompeo A, Beznoussenko G, Castaldi C, Cortellino S, McNiven MA, Luini A, Buccione R (2003). Dynamin participates in focal extracellular matrix degradation by invasive cells. Mol Biol Cell 14: 1074-1084.

Beaty BT, Sharma VP, Bravo-Cordero JJ, Simpson MA, Eddy RJ, Koleske AJ, Condeelis J (2013). $\beta 1$ integrin regulates Arg to promote invadopodial maturation and matrix degradation. Mol Biol Cell 24: 1661-1675. can we better exploit upstream regulators and structural components of invadopodia for the diagnosis and therapy of cancer?

\section{Glossary}

Cortactin: Cortical actin binding protein. Cortactin promotes actin polymerization.

Cofilin: An actin binding protein. Cofilin severs actin filaments.

N-WASP: Neural Wiskott-Aldrich syndrome protein. $\mathrm{N}$-WASP promotes actin nucleation.

Tks5: Tyrosine kinase substrate 5 , a scaffold protein.

Nck1: Noncatalytic region of tyrosine kinase adaptor protein 1 . Nck1 is an adaptor protein involved in signal transduction.

MT1-MMP: MT1-MMP is a matrix metalloproteinase also known as MMP-14.

Src: The first described proto-oncogene. A nonreceptor tyrosine kinase.

Arg: A member of the Abelson family of nonreceptor tyrosine kinases.

NHE1: A Na+/H+ exchanger.

RhoC: A member of the Rho family of small GTPases.

\section{Acknowledgments}

The authors gratefully acknowledge funding support from the Scientific and Technological Research Council of Turkey (Project no: 111T547).

Blouw B, Seals DF, Pass I, Diaz B, Courtneidge SA (2008). A role for the podosome/invadopodia scaffold protein Tks5 in tumor growth in vivo. Eur J Cell Biol 87: 555-567.

Bowden ET, Barth M, Thomas D, Glazer RI, Mueller SC (1999). An invasion-related complex of cortactin, paxillin and $\mathrm{PKCmu}$ associates with invadopodia at sites of extracellular matrix degradation. Oncogene 18: 4440-4449.

Bowden ET, Onikoyi E, Slack R, Myoui A, Yoneda T, Yamada KM, Mueller SC (2006). Co-localization of cortactin and phosphotyrosine identifies active invadopodia in human breast cancer cells. Exp Cell Biol 312: 1240-1253.

Bravo-Cordero JJ, Hodgson L, Condeelis J (2012). Directed cell invasion and migration during metastasis. Curr Opin Cell Biol 24: 277-283.

Bravo-Cordero JJ, Oser M, Chen XM, Eddy R, Hodgson L, Condeelis J (2011). A Novel spatiotemporal RhoC activation pathway locally regulates cofilin activity at invadopodia. Curr Biol 21: 635-644.

Buccione R, Caldieri G, Ayala I (2009). Invadopodia: specialized tumor cell structures for the focal degradation of the extracellular matrix. Cancer Metast Rev 28: 137-149.

Buday L, Downward J (2007). Roles of cortactin in tumor pathogenesis. Bba-Rev Cancer 1775: 263-273. 
Caldieri G, Ayala I, Attanasio F, Buccione R (2009). Cell and molecular biology of invadopodia. Int Rev Cell Mol Biol 275: 1-34.

Caldieri G, Buccione R (2010). Aiming for invadopodia: organizing polarized delivery at sites of invasion. Trends Cell Biol 20: 6470 .

Carpenter G, Cohen S (1990). Epidermal growth-factor. J Biol Chem 265: 7709-7712.

Chen WT (1996). Proteases associated with invadopodia, and their role in degradation of extracellular matrix. Enzyme Protein 49: 59-71.

Chen WT, Lee CC, Goldstein L, Bernier S, Liu CHL, Lin CY, Yeh YY, Monsky WL, Kelly T, Dai MZ et al. (1994). Membrane proteases as potential diagnostic and therapeutic targets for breast malignancy. Breast Cancer Res Tr 31: 217-226.

Clark ES, Brown B, Whigham AS, Kochaishvili A, Yarbrough WG, Weaver AM (2009). Aggressiveness of HNSCC tumors depends on expression levels of cortactin, a gene in the 11q13 amplicon. Oncogene 28: 431-444.

Cohen S (1962). Isolation of a mouse submaxillary gland protein accelerating incisor eruption and eyelid opening in the newborn animal. J Biol Chem 237: 1555-1562.

DesMarais V, Yamaguchi H, Oser M, Soon L, Mouneimne G, Sarmiento C, Eddy R, Condeelis J (2009). N-WASP and cortactin are involved in invadopodium-dependent chemotaxis to EGF in breast tumor cells. Cell Motil Cytoskel 66: 303-316.

Destaing O, Block MR, Planus E, Albiges-Rizo C (2011). Invadosome regulation by adhesion signaling. Curr Opin Cell Biol 23: 597606.

Gil-Henn H, Patsialou A, Wang Y, Warren MS, Condeelis JS, Koleske AJ (2013). Arg/Abl2 promotes invasion and attenuates proliferation of breast cancer in vivo. Oncogene 32: 2622-2630.

Gilcrease MZ (2007). Integrin signaling in epithelial cells. Cancer Lett 247: 1-25.

Gimona M, Buccione R (2006). Adhesions that mediate invasion. Int J Biochem Cell B 38: 1875-1892.

Gimona M, Buccione R, Courtneidge SA, Linder S (2008). Assembly and biological role of podosomes and invadopodia. Curr Opin Cell Biol 20: 235-241.

Gligorijevic B, Wyckoff J, Yamaguchi H, Wang YR, Roussos ET, Condeelis J (2012). N-WASP-mediated invadopodium formation is involved in intravasation and lung metastasis of mammary tumors. J Cell Sci 125: 724-734.

Harris RC, Chung E, Coffey RJ (2003). EGF receptor ligands. Exp Cell Biol 284: 2-13.

Hoshino D, Branch KM, Weaver AM (2013). Signaling inputs to invadopodia and podosomes. J Cell Sci 126: 2979-2989.

Kessenbrock K, Plaks V, Werb Z (2010). Matrix metalloproteinases: regulators of the tumor microenvironment. Cell 141: 52-67.

Labernadie A, Thibault C, Vieu C, Maridonneau-Parini I, Charriere GM (2010). Dynamics of podosome stiffness revealed by atomic force microscopy. P Natl Acad Sci USA 107: 21016-21021.
Leight JL, Alge DL, Maier AJ, Anseth KS (2013). Direct measurement of matrix metalloproteinase activity in $3 \mathrm{D}$ cellular microenvironments using a fluorogenic peptide substrate. Biomaterials 34: 7344-7352.

Lewis S, Locker A, Todd JH, Bell JA, Nicholson R, Elston CW, Blamey RW, Ellis IO (1990). Expression of epidermal growth-factor receptor in breast carcinoma. J Clin Pathol 43: 385-389.

Li A, Dawson JC, Forero-Vargas M, Spence HJ, Yu XZ, Konig I, Anderson K, Machesky LM (2010a). The actin-bundling protein fascin stabilizes actin in invadopodia and potentiates protrusive invasion. Curr Biol 20: 339-345.

Li RH, Li G, Deng L, Liu QL, Dai J, Shen J, Zhang J (2010b). IL-6 augments the invasiveness of U87MG human glioblastoma multiforme cells via up-regulation of MMP-2 and fascin-1. Oncol Rep 23: 1553-1559.

Linder S (2007). The matrix corroded: podosomes and invadopodia in extracellular matrix degradation. Trends Cell Biol 17: 107-117.

Linder S (2009). Invadosomes at a glance. J Cell Sci 122: 3009-3013.

Linder S, Aepfelbacher M (2003). Podosomes: adhesion hot-spots of invasive cells. Trends Cell Biol 13: 376-385.

Linder S, Wiesner C, Himmel M (2011). Degrading devices: invadosomes in proteolytic cell invasion. Annu Rev Cell Dev Biol 27: 185-211.

Lizarraga F, Poincloux R, Romao M, Montagnac G, Le Dez G, Bonne I, Rigaill G, Raposo G, Chavrier P (2009). Diaphanous-related formins are required for invadopodia formation and invasion of breast tumor cells. Cancer Res 69: 2792-2800.

MacGrath SM, Koleske AJ (2012). Cortactin in cell migration and cancer at a glance. J Cell Sci 125: 1621-1626.

Mader CC, Oser M, Magalhaes MAO, Bravo-Cordero JJ, Condeelis J, Koleske AJ, Gil-Henn H (2011). An EGFR-Src-Arg-cortactin pathway mediates functional maturation of invadopodia and breast cancer cell invasion. Cancer Res 71: 1730-1741.

Magalhaes MAO, Larson DR, Mader CC, Bravo-Cordero JJ, Gil-Henn H, Oser M, Chen XM, Koleske AJ, Condeelis J (2011). Cortactin phosphorylation regulates cell invasion through a $\mathrm{pH}$-dependent pathway. J Cell Biol 195: 903-920.

Massague J, Pandiella A (1993). Membrane-anchored growth factors. Annu Rev Biochem 62: 515-541.

McNiven MA (2013). Breaking away: matrix remodeling from the leading edge. Trends Cell Biol 23: 16-21.

Memon AA, Sorensen BS, Nexo E (2006). The epidermal growth factor family has a dual role in deciding the fate of cancer cells. Scand J Clin Lab Inv 66: 623-630.

Moro L, Dolce L, Cabodi S, Bergatto E, Erba EB, Smeriglio M, Turco E, Retta SF, Giuffrida MG, Venturino M et al. (2002). Integrininduced epidermal growth factor (EGF) receptor activation requires $\mathrm{c}$-Src and p130Cas and leads to phosphorylation of specific EGF receptor tyrosines. J Biol Chem 277: 9405-9414.

Mueller SC, Ghersi G, Akiyama SK, Sang QXA, Howard L, PineiroSanchez M, Nakahara H, Yeh Y, Chen WT (1999). A novel protease-docking function of integrin at invadopodia. J Biol Chem 274: 24947-24952. 
Murphy DA, Courtneidge SA (2011). The 'ins' and 'outs' of podosomes and invadopodia: characteristics, formation and function. Nat Rev Mol Cell Bio 12: 413-426.

Oser M, Dovas A, Cox D, Condeelis J (2011). Nck1 and Grb2 localization patterns can distinguish invadopodia from podosomes. Eur J Cell Biol 90: 181-188.

Oser M, Mader CC, Gil-Henn H, Magalhaes M, Bravo-Cordero JJ, Koleske AJ, Condeelis J (2010). Specific tyrosine phosphorylation sites on cortactin regulate Nck1-dependent actin polymerization in invadopodia. J Cell Sci 123:3662-3673.

Oser M, Yamaguchi H, Mader CC, Bravo-Cordero JJ, Arias M, Chen XM, DesMarais V, van Rheenen J, Koleske AJ, Condeelis J (2009). Cortactin regulates cofilin and N-WASp activities to control the stages of invadopodium assembly and maturation. J Cell Biol 186: 571-587.

Packard BZ, Artym VV, Komoriya A, Yamada KM (2009). Direct visualization of protease activity on cells migrating in threedimensions. Matrix Biol 28: 3-10.

Ruoslahti E, Yamaguchi Y, Hildebrand A, Border WA (1992). Extracellular-matrix growth-factor interactions. Cold Spring Harb Sym 57: 309-315.

Sabeh F, Shimizu-Hirota R, Weiss SJ (2009). Protease-dependent versus -independent cancer cell invasion programs: threedimensional amoeboid movement revisited. J Cell Biol 185: 11-19.

Sainsbury JRC, Malcolm AJ, Appleton DR, Farndon JR, Harris AL (1985). Presence of epidermal growth-factor receptor as an indicator of poor prognosis in patients with breast cancer. J Clin Pathol 38: 1225-1228.

Schoumacher M, Goldman RD, Louvard D, Vignjevic DM (2010). Actin, microtubules, and vimentin intermediate filaments cooperate for elongation of invadopodia. J Cell Biol 189: 541556.

Seals DF, Azucena EF, Pass I, Tesfay L, Gordon R, Woodrow M, Resau JH, Courtneidge SA (2005). The adaptor protein Tks5/ Fish is required for podosome formation and function, and for the protease-driven invasion of cancer cells. Cancer Cell 7: 155-165.

Sharma VP, Eddy R, Entenberg D, Kai M, Gertler FB, Condeelis J (2013). Tks5 and SHIP2 regulate invadopodium maturation, but not initiation, in breast carcinoma cells. Curr Biol 23: 2079-2089.
Singh AB, Harris RC (2005). Autocrine, paracrine and juxtacrine signaling by EGFR ligands. Cell Signal 17: 1183-1193.

Stylli SS, Kaye AH, Lock P (2008). Invadopodia: At the cutting edge of tumour invasion. J Clin Neurosci 15: 725-737.

Stylli SS, Stacey TTI, Kaye AH, Lock P (2012). Prognostic significance of Tks5 expression in gliomas. J Clin Neurosci 19: 436-442.

Taipale J, Keski-Oja J (1997). Growth factors in the extracellular matrix. Faseb J 11: 51-59.

Thery M, Racine V, Piel M, Pepin A, Dimitrov A, Chen Y, Sibarita JB, Bornens M (2006). Anisotropy of cell adhesive microenvironment governs cell internal organization and orientation of polarity. P Natl Acad Sci USA 103: 19771-19776.

Wang WG, Goswami S, Lapidus K, Wells AL, Wyckoff JB, Sahai E, Singer RH, Segall JE, Condeelis JS (2004). Identification and testing of a gene expression signature of invasive carcinoma cells within primary mammary tumors. Cancer Res 64: 85858594.

Wang WG, Wyckoff JB, Goswami S, Wang YR, Sidani M, Segall JE, Condeelis JS (2007). Coordinated regulation of pathways for enhanced cell motility and chemotaxis is conserved in rat and mouse mammary tumors. Cancer Res 67: 3505-3511.

Yamada KM, Even-Ram S (2002). Integrin regulation of growth factor receptors. Nat Cell Biol 4: E75-E76.

Yamaguchi H (2012). Pathological roles of invadopodia in cancer invasion and metastasis. Eur J Cell Biol 91: 902-907.

Yamaguchi H, Lorenz M, Kempiak S, Sarmiento C, Coniglio S, Symons M, Segall J, Eddy R, Miki H, Takenawa T et al. (2005). Molecular mechanisms of invadopodium formation: the role of the N-WASP-Arp2/3 complex pathway and cofilin. J Cell Biol 168: 441-452.

Yilmaz M, Christofori G (2009). EMT, the cytoskeleton, and cancer cell invasion. Cancer Metast Rev 28: 15-33.

Yu XZ, Machesky LM (2012). Cells assemble invadopodia-like structures and invade into Matrigel in a matrix metalloprotease dependent manner in the circular invasion assay. Plos One 7.

Yu XZ, Zech T, McDonald L, Gonzalez EG, Li A, Macpherson I, Schwarz JP, Spence H, Futo K, Timpson P et al. (2012). $\mathrm{N}$-WASP coordinates the delivery and F-actin-mediated capture of MT1-MMP at invasive pseudopods. J Cell Biol 199: 527-544. 